\title{
Scientific assessment of the quality of OSIRIS images
}

C. Tubiana ${ }^{1}$, C. Güttler ${ }^{1}$, G. Kovacs ${ }^{1}$, I. Bertini ${ }^{2}$, D. Bodewits ${ }^{3}$, S. Fornasier ${ }^{4}$, L. Lara ${ }^{5}$, F. La Forgia ${ }^{2}$, S. Magrin ${ }^{6}$, M. Pajola ${ }^{2}$, H. Sierks ${ }^{1}$, C. Barbieri ${ }^{7}$, P. L. Lamy ${ }^{8}$, R. Rodrigo ${ }^{9}, 10$, D. Koschny ${ }^{11}$, H. Rickman ${ }^{12,13}$, H. U. Keller ${ }^{15,14}$, J. Agarwal ${ }^{1}$, M. F. A'Hearn ${ }^{3}$, M. A. Barucci ${ }^{4}$, J.-L. Bertaux ${ }^{16}$, S. Besse ${ }^{2}$, S. Boudreault ${ }^{1}$, G. Cremonese ${ }^{17}$, V. Da Deppo ${ }^{18}$, B. Davidsson ${ }^{12}$, S. Debei ${ }^{19}$, M. De $\mathrm{Cecco}^{20}$, M. R. El-Maarry ${ }^{21}$, M. Fulle ${ }^{22}$, O. Groussin ${ }^{8}$, P. Gutiérrez-Marques ${ }^{1}$, P. J. Gutiérrez ${ }^{5}$, N. Hoekzema ${ }^{1}$, M. Hofmann ${ }^{1}$, S. F. Hviid ${ }^{14,1}$, W.-H. Ip ${ }^{23,24}$, L. Jorda ${ }^{8}$, J. Knollenberg ${ }^{14}$, J.-R. Kramm ${ }^{1}$, E. Kührt ${ }^{14}$, M. Küppers ${ }^{25}$, M. Lazzarin ${ }^{7}$, J. J. Lopez Moreno ${ }^{5}$, F. Marzari ${ }^{7}$, M. Massironi2 ${ }^{2,26}$, H. Michalik ${ }^{27}$, R. Moissl ${ }^{25}$, G. Naletto ${ }^{28,2,18}$, N. Oklay ${ }^{1}$, F. Scholten ${ }^{14}$, X. Shi ${ }^{1}$, N. Thomas ${ }^{21,29}$, and J.-B. Vincent ${ }^{1}$

${ }^{1}$ Max-Planck Institut für Sonnensystemforschung, Justus-von-Liebig-Weg, 237077 Göttingen, Germany. e-mail: tubiana@mps .mpg.de

2 Centro di Ateneo di Studi ed Attivita Spaziali “Giuseppe Colombo" (CISAS), University of Padova, via Venezia 15, 35131 Padova, Italy

3 Department of Astronomy, University of Maryland, College Park, MD 20742-2421, USA

${ }^{4}$ LESIA-Observatoire de Paris, CNRS, Université Pierre et Marie Curie, Université Paris Diderot, 5, Place J. Janssen, 92195 Meudon Principal Cedex, France

5 Instituto de Astrofísica de Andalucía (CSIC), c/ Glorieta de la Astronomia s/n, 18008 Granada, Spain

6 Dipartimento di Fisica ed Astronomia, Università di Padova, via Marzolo 8, 35131 Padova, Italy

7 University of Padova, Department of Physics and Astronomy, Vicolo dell' Osservatorio 3, 35122 Padova, Italy

8 Aix Marseille Université, CNRS, LAM (Laboratoire d'Astrophysique de Marseille) UMR 7326, 13388 Marseille, France

9 Centro de Astrobiologia, CSIC-INTA, 28850 Torrejon de Ardoz, Madrid, Spain

10 International Space Science Institute, Hallerstrasse 6, 3012 Bern, Switzerland

11 Scientific Support Office, European Space Research and Technology Centre/ESA, Keplerlaan 1, Postbus 299, 2201 AZ Noordwijk ZH, The Netherlands

12 Department of Physics and Astronomy, Uppsala University, Box 516, 75120 Uppsala, Sweden

13 PAS Space Research Center, Bartycka 18A, 00716 Warszawa, Poland

14 Deutsches Zentrum für Luft- und Raumfahrt (DLR), Institut für Planetenforschung, Rutherfordstrsse 2, 12489 Berlin, Germany

15 Institut für Geophysik und extraterrestrische Physik (IGEP), Technische Universität Braunschweig, Mendelssohnstr. 3, 38106 Braunschweig, Germany

16 LATMOS, CNRS/UVSQ/IPSL, 11 boulevard d'Alembert, 78280 Guyancourt, France

17 INAF, Osservatorio Astronomico di Padova, Vicolo dell'Osservatorio 5, 35122 Padova, Italy

18 CNR-IFN UOS Padova LUXOR, via Trasea 7, 35131 Padova, Italy

19 Department of Industrial Engineering, University of Padova, via Venezia 1, 35131 Padova, Italy

20 University of Trento, via Sommarive 9, 38123 Trento, Italy

21 Physikalisches Institut der Universität Bern, Sidlerstr. 5, 3012 Bern, Switzerland

22 INAF-Osservatorio Astronomico di Trieste, via Tiepolo 11, 34014 Trieste, Italy

23 Graduate Institute of Astronomy, National Central University, 300 Chung-Da Rd, 32054 Chung-Li, Taiwan

24 Space Science Institute, Macau University of Science and Technology, Macao, PR China

25 Operations Department, European Space Astronomy Centre/ESA, PO Box 78, 28691 Villanueva de la Cañada (Madrid), Spain

26 Dipartimento di Geoscienze, University of Padova, via Gradenigo 6, 35131 Padova, Italy

27 Institut für Datentechnik und Kommunikationsnetze der TU Braunschweig, Hans-Sommer-Str. 66, 38106 Braunschweig, Germany

28 Department of Information Engineering, University of Padova, via Gradenigo 6/B, 35131 Padova, Italy

29 Center for Space and Habitability, University of Bern, 3012 Bern, Switzerland

Received 27 February 2015 / Accepted 11 August 2015

\section{ABSTRACT}

Context. OSIRIS, the scientific imaging system onboard the ESA Rosetta spacecraft, has been imaging the nucleus of comet 67P/Churyumov-Gerasimenko and its dust and gas environment since March 2014. The images serve different scientific goals, from morphology and composition studies of the nucleus surface, to the motion and trajectories of dust grains, the general structure of the dust coma, the morphology and intensity of jets, gas distribution, mass loss, and dust and gas production rates.

Aims. We present the calibration of the raw images taken by OSIRIS and address the accuracy that we can expect in our scientific results based on the accuracy of the calibration steps that we have performed.

Methods. We describe the pipeline that has been developed to automatically calibrate the OSIRIS images. Through a series of steps, radiometrically calibrated and distortion corrected images are produced and can be used for scientific studies. Calibration campaigns were run on the ground before launch and throughout the years in flight to determine the parameters that are used to calibrate the images and to verify their evolution with time. We describe how these parameters were determined and we address their accuracy. Results. We provide a guideline to the level of trust that can be put into the various studies performed with OSIRIS images, based on the accuracy of the image calibration.

Key words. instrumentation: detectors - methods: data analysis - space vehicles: instruments 


\section{Introduction}

Launched in 2004, the Rosetta spacecraft woke up on January 20, 2014, after a ten-year cruise and 30 months of deep space hibernation. The Optical, Spectroscopic, and Infrared Remote Imaging System (OSIRIS; Keller et al. 2007) is the scientific camera system onboard Rosetta. It comprises a Narrow Angle Camera (NAC) and a Wide Angle Camera (WAC) with a field of view (FOV) of $2.20^{\circ} \times 2.22^{\circ}$ and $11.35^{\circ} \times 12.11^{\circ}$, respectively, and an instantaneous field of view (IFOV) of $18.6 \mu \mathrm{rad} / \mathrm{pix}$ and of $101.0 \mu \mathrm{rad} / \mathrm{pix}$, respectively. Both cameras use a $2048 \times 2048$ pixel backside illuminated CCD detector with a UV optimized anti-reflection coating. The CCDs are equipped with lateral anti-blooming that allows overexposure of the nucleus without creating saturation artifacts, enabling the study of details in the faint coma structures next to the illuminated limb.

The CCD full well capacity is $>120000 \mathrm{e}^{-} /$pix (Keller et al. 2007). The pixel linearity is guaranteed only below this limit. A gain value of $3.1 \mathrm{e}^{-} / \mathrm{DN}$ ( $\mathrm{DN}=$ digital number) in $\mathrm{HIGH}$ gain mode and $15.5 \mathrm{e}^{-} / \mathrm{DN}$ in LOW gain mode is used, as specified by the manufacturer. Calibration images to measure the gain were acquired in December 2014, and will be used to investigate whether an update to the current gain values is needed. The NAC and WAC are equipped with two readout amplifier each that can be used independently or together to achieve a faster readout of the image. Both cameras are off-axis systems, with no central obscuration along the beam. The off-axis design has the advantage of providing high transmittance from the UV to the near-IR and diffraction limited performance with low geometrical optical aberration, but introduces a significant geometric distortion that needs to be corrected for scientific use of the image products.

The NAC is equipped with 11 filters covering a wavelength range of 250-1000 nm, while the WAC has 14 filters covering a range of 240-720 nm. Figure 1 shows the transmission curves of the NAC and WAC filters.

In addition to the bandpass filters, the NAC filter wheels contain a neutral density filter and anti-reflection coated focus plates: three far focus plates (FFP-UV, FFP-VIS, and FFP-IR) and a near focus plate (NFP-VIS). The focus plates, combined with the bandpass filters, allow two focusing ranges: far focus from infinity to $2 \mathrm{~km}$, optimized at $4 \mathrm{~km}$, and near focus from $2 \mathrm{~km}$ to $1 \mathrm{~km}$, optimized at $1.3 \mathrm{~km}$. Both cameras have a planeparallel $12 \mathrm{~mm}$ thick anti-reflection coated plate (ARP) in front of the CCD for radiation shielding. The transmission curves of the focus plates, the anti-reflection coated plates, together with the total reflectivity of the mirror system and the quantum efficiency of the CCD are plotted in Fig. 1.

The NAC and WAC have been designed as a complementary pair that addresses, on the one hand, the study of the nucleus surface, and, on the other hand, the investigation of the dynamics of the sublimation processes. The NAC, with its high spatial resolution, was used to detect the nucleus of 67P from a distance of millions of kilometers, and it is now used to study the morphology and mineralogy of the surface and details of the dust ejection process. The WAC has a lower spatial resolution and, accordingly, a much wider field of view. This allows observations of the 3D flow-field of dust and gas even if the spacecraft is near the nucleus and provides a synoptic view of the nucleus for context of the NAC and other instruments onboard Rosetta. To summarize, the WAC provides long-term monitoring of the entire nucleus and its surrounding, while the NAC studies the surface details.
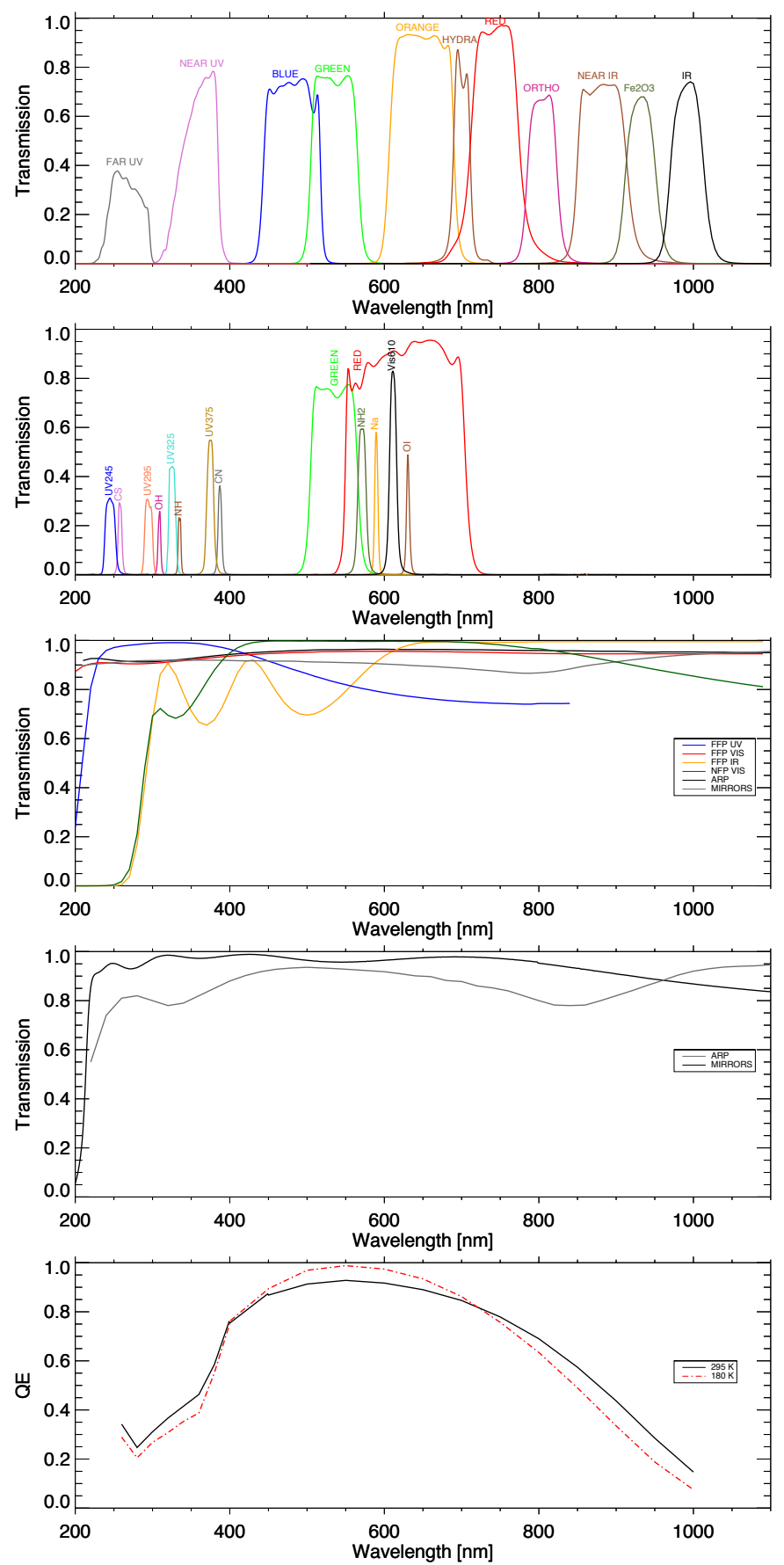

Fig. 1. Transmission curves of the NAC (first panel) and WAC (second panel) filters. The third panel shows the transmission curves of the NAC focus plates, the anti-reflection coated plates, together with the total reflectivity of the NAC mirror system. The transmission curve of the WAC anti-reflection coated plate and the total reflectivity of the WAC mirror system is shown in the fourth panel. The last panel shows the quantum efficiency of the NAC CCD as measured on the flight model at room temperature (black solid line) and close to operational temperature (red dot-dashed line).

\section{Scientific rationale for calibrating OSIRIS images}

The images acquired by OSIRIS serve different scientific goals, which are mostly but certainly not comprehensively (i) shape reconstruction of the nucleus and morphology of the surface; (ii) composition (color) of the nucleus and the dust; (iii) motion and trajectories of dust grains; (iv) general structure of the dust 
coma; (v) morphology and intensity of jets; and (vi) gas distribution and production rates. The acquired images are calibrated with the OSIRIS calibration pipeline (as described in Sect. 3). To explain the scientific need for the best possible image calibration, this section addresses the calibration steps that are important when preparing the data for the studies quoted above.

Shape reconstruction. After the post-hibernation recommissioning, the OSIRIS cameras were used to observe the nucleus of $67 \mathrm{P}$ on a regular basis in order to retrieve its rotational parameters and its shape as early as possible. These sequences, acquired when Rosetta was approaching the comet, were followed by detailed observations of the surface during the global mapping phase. These images have been used by photoclinometry, photogrammetry, and stereo landmarks tracking software to retrieve the actual shape and the direction of the spin axis of the comet with an increasing accuracy. All these techniques either use images corrected for the geometric distortion of the camera or must account for the geometric camera model during the calculation. Photoclinometry techniques also depend on the radiometric correction to reconstruct slopes from pixel-to-pixel variations detected in the images.

Geomorphology. Images of the nucleus surface are used for various geomorphological observations and geological interpretations, which include morphology-based region definitions (Thomas et al. 2015; El-Maarry et al. 2015), localized geomorphological investigations (Auger et al. 2015), boulder counting and analysis (Pajola et al. 2015; Pommerol et al. 2015), and geostructural studies (Massironi et al. 2015). An accurate geometric distortion correction is essential to all these studies to accurately locate and measure all kinds of features in various images. Indeed, quantitative geomorphological and geo-structural investigations are practically impossible with geometrically distorted images that need to be inserted into geographic information system (GIS) software possibly overlapping distortionfree images on shape models and digital terrain models (DTMs). Indeed, quantitative geomorphological and geo-structural investigations are practically impossible with geometrically distorted images that need to be inserted into geographic information system (GIS) software possibly overlapping distortion-free images on shape models and digital terrain models (DTMs).

Composition of the nucleus. Bandpass filters covering the wavelength range from near-UV to IR are well suited for global and local studies of the nucleus photometric properties and spectrophotometry (Fornasier et al. 2015). The aim is to investigate the heterogeneity of the nucleus surface in terms of composition and albedo at several scales. The radiometric calibration plays a key role in the compositional studies, and geometric distortion correction is important to properly co-register images to create color ratios.

Dust coma. A dust coma around the nucleus of 67P has been observed in OSIRIS images since the pronounced outburst that occurred between April 27 and 30, 2014, or even earlier (Tubiana et al. 2015). The study of the overall dust coma, its variations with time and heliocentric distance require, as in the case of gas studies, an accurate bias subtraction and a good understanding of the dark current. The radiometric calibration is essential in order to determine dust production rates from the dust flux measured in the images.

Dust aggregates, snowballs, and grains. Multicolor NAC and WAC images of the inner coma have been obtained since August 4, 2014, to monitor the production and the motion of large chunks of material ejected by the increasing cometary activity while $67 \mathrm{P}$ is approaching perihelion. The geometric distortion correction and a precise radiometric calibration are mandatory to study the nature of these objects, their lifetimes in the inner coma, and the connection with the complex gravitational field of 67P. NAC images acquired on August 4, 2014 (Rotundi et al. 2015), and WAC images acquired on September 10, 2014 (Davidsson et al. 2015), show a large number of individual grains. Radiometrically calibrated and distortion corrected images are used to determine the trajectories of the grains and to measure their intensities and colors. Colors provide hints about the grain composition and comparing grain and nucleus colors we can investigate from which region on the nucleus the grains were emitted.

Dust phase function. Multicolor WAC images have been taken since January 9, 2015, to study the multi-wavelength phase function of unresolved cometary dust in the coma. Also in this context, the distortion correction and the radiometric calibration are important in order to reconstruct the precise 3D geometry of the pyramidal FOV and to obtain precise flux measurements. Combining these factors, we obtain the albedo versus phase angle curve that can then be compared with theoretical models of cometary dust (Bertini et al. 2007) to derive detailed information on the physical nature of tiny dust particles emitted from the nucleus.

Search for satellites. NAC images were acquired on July 20, 2014, when Rosetta was approaching 67P, to search for large objects in the vicinity of the comet's nucleus both for scientific and spacecraft security issues (Bertini et al. 2015). The geometric distortion correction is fundamental to properly identifying the stellar background in order to avoid spurious detections. At the same time, the radiometric calibration allows the measure of limiting fluxes and the consequent determination of limiting sizes of the objects.

Optical thickness of overall dust coma and jets. Background stars are serendipitously present in many OSIRIS images obtained while the spacecraft is orbiting the comet. In addition, several specific image series are planned to follow the motion of stars within the projected coma due to the spacecraft motion. Both radiometric calibration and distortion correction are necessary to precisely locate the OSIRIS-star line of sight and determine the magnitude of the light source. This allows us to obtain measurements of the coma column density versus the nucleus distance and its optical thickness. These observations can provide direct measurements of density and dust albedo (Lacerda \& Jewitt 2012).

Gas distribution and production rates. The WAC is equipped with a set of narrowband filters to study cometary gas emissions of $\mathrm{CS}, \mathrm{OH}, \mathrm{NH}, \mathrm{CN}, \mathrm{NH}_{2}, \mathrm{Na}$, and $\mathrm{OI}$ (see Fig. 1). The resulting gas surface brightness is low $(S / N \sim 5-100$ in continuum subtracted images), thus an accurate bias subtraction is crucial. In this case, even the small temperature dependence of the bias becomes important when it is similar to the signal level. The narrowband filter images contain both gas emission lines and the reflected continuum of the omnipresent dust such that an accurate dust continuum removal is needed to measure the gas signal. We empirically determined the dust continuum removal factor using 16Cyg A+B, which are not spatially resolved in WAC images. These factors are adjusted for the reddening of the dust. A careful radiometric calibration is fundamental to accurately measure the gas surface brightness and therefore determine the gas column densities, which are then modeled to derive the gas production rate.

For both dust coma and gas studies, where the dust and gas surface brightness are not very strong, a major problem is the presence of ghosts and stray light in the images. Their removal is not currently implemented in the calibration pipeline 
and the treatment of these issues is beyond the scope of this work. Additional care should be taken when analyzing gas and dust images.

Jets. Since the end of July 2014, images of 67P have shown a large number of jets arsing from the surface of the comet (Lin et al. 2015), with a signal of 10-30\% above the general coma background. To study their morphology, their evolution with time, and their origins on the surface a proper geometric distortion correction has to be applied to the images. Studies of photometric profiles along the jets and thus density variations with distance from the surface, require precise radiometric calibration.

\section{The OSIRIS calibration pipeline}

A series of steps is needed to go from the acquired images to the calibrated ones that can be used for a quantitative data analysis. Data are downloaded from the spacecraft memory, raw images (level 0) are assembled from the data packets, and the actual instrument hardware parameters are decoded. From these data, raw images with calibrated hardware parameters and spacecraft pointing information (level 1) are generated. Those steps are handled by the OSIRIS software package OsiTrap, and its description is not part of this paper. Raw images are then calibrated by another software called OsiCalliope, which is described in the following paragraphs.

OsiCalliope, the OSIRIS scientific calibration pipeline, is a complex software package that converts the raw CCD images (level 1) to radiometrically calibrated and geometric distortion corrected images (level 2 and 3, respectively) through a series of intermediate steps shown in the flowchart in Fig. 2.

Calibration campaigns were run on the ground before launch and in flight to determine each of the parameters that is used by OsiCalliope to calibrate the OSIRIS images and to verify their behavior over time. In May 2014, about a month after OSIRIS was recommissioned after 30 months of hibernation, an extensive calibration campaign was carried out to determine up-todate calibration parameters and to assess how the instrument is performing after 10 years in flight.

Hereafter, we describe each calibration step performed by OsiCalliope and how and when each of the calibration parameters was determined.

Correction of the tandem ADC offset. The NAC and the WAC are each equipped with two 14-bit analog-to-digital converters (ADCs) for each readout channel to digitize the CCD pixel signal. The readout electronics can use the two ADCs separately or coupled together in a dual 14-bit ADC configuration (ADCTANDEM, which is used for most images), achieving a dynamical range of almost 16 bits. The two coupled ADCs have a fixed amplification ratio setting of 4 . The ADC-TANDEM configuration provides full 14-bit resolution in the range $0-2^{14}-1$, while in the range $2^{14}-2^{16}-1$ the last two bits are always zero. When in ADC-TANDEM configuration, the two ADCs are adjusted to cover a continuous linear range, although an offset of a few DN is present and is removed by the pipeline. This constant offset has been determined during the camera assembly (Keller et al. 2007) and it is assumed to be constant. It cannot be re-measured on the flight unit with the needed accuracy. However, linearity tests performed using Vega observations would show if the offset is deviating from the currently used values.

Bias subtraction. Analysis of the bias frames acquired over the years indicates that the bias is constant throughout the CCD (no significant pixel-to-pixel variations) for a given operational mode and CCD temperature. The bias is therefore removed as a constant number from all pixels in a given image.

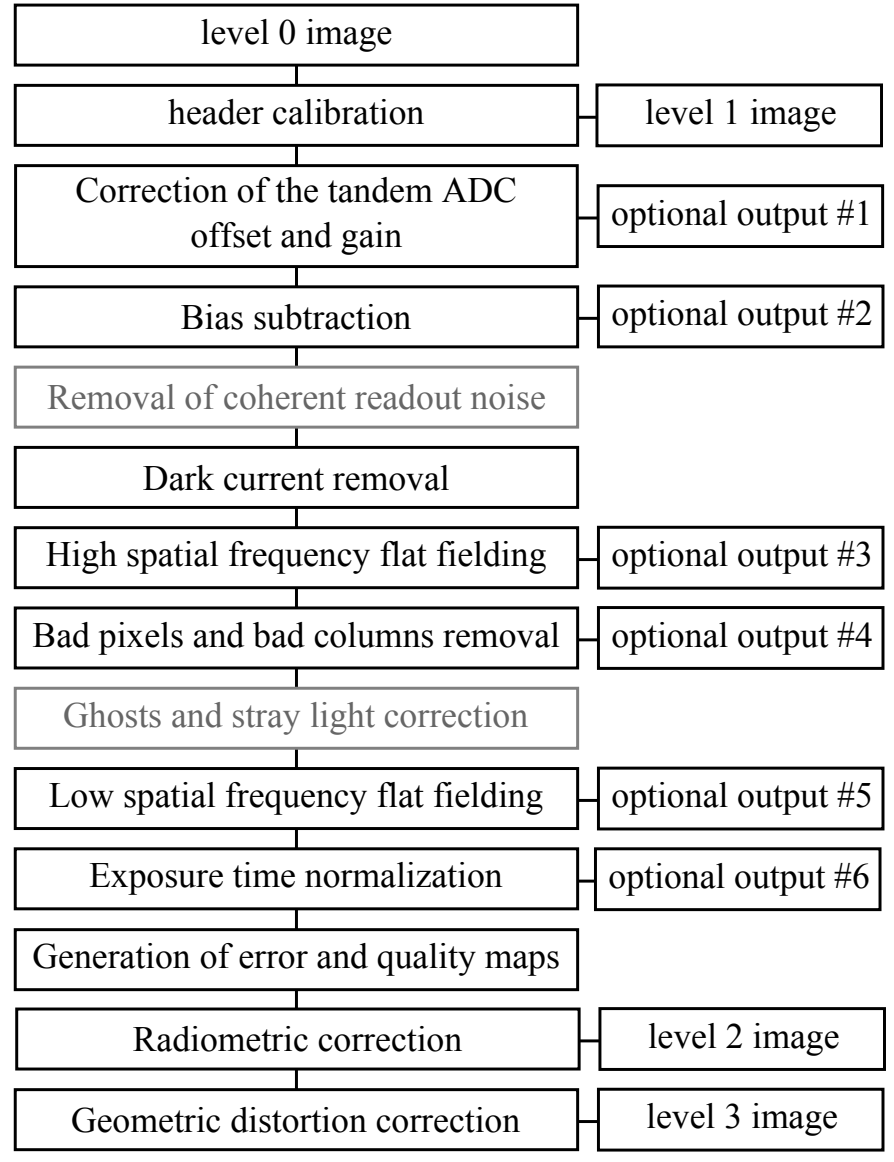

Fig. 2. Flowchart of the OSIRIS calibration pipeline. Gray boxes indicate steps that are not implemented in the current version of the pipeline.

The bias is constant for all software windowing and binning modes, but changes with the hardware window size, binning, and amplifier channel used. One bias value is determined for each operational mode. The bias is also found to be correlated with the ADC temperature; an additional correction to the data is applied to correct for this effect.

To determine the bias level to be subtracted from the images we use a $3 \sigma$ filter method. It consists in discarding pixels with count above a certain threshold, i.e., $3 \sigma$, from the average of all pixels in the $\mathrm{CCD}$, to remove hot pixels and cosmic rays from the bias determination. This is achieved in an iterative way, discarding first pixels with counts above $10 \sigma$ from the average, then $5 \sigma$ from the new average, and finally $3 \sigma$ from the third average. The average and standard deviation of pixels with intensity below the $3 \sigma$ threshold are the bias and corresponding uncertainty used to calibrate the images. The bias determination has an accuracy (at the $1 \sigma$ level) of $2-4 \%$ relative to a typical value of $230 \mathrm{DN}$. The bias has a much higher intrinsic accuracy given by the average over the number of pixels. However, the contribution from the readout noise and the coherent noise cannot be disentangled, increasing the uncertainty on the bias determination.

We have analyzed the dependence of the bias level over the ADC temperature for the nominal configurations and obtained that it is on the order of $0.5-0.7 \mathrm{DN} / \mathrm{K}$. The temperature variation since OSIRIS switched on in March 2014 is on the order of $5 \mathrm{~K}$, which translates to a maximum bias correction of 3.5 DN. Rosetta is getting closer to the Sun and the ADC temperature will rise as a consequence of the increasing temperature 


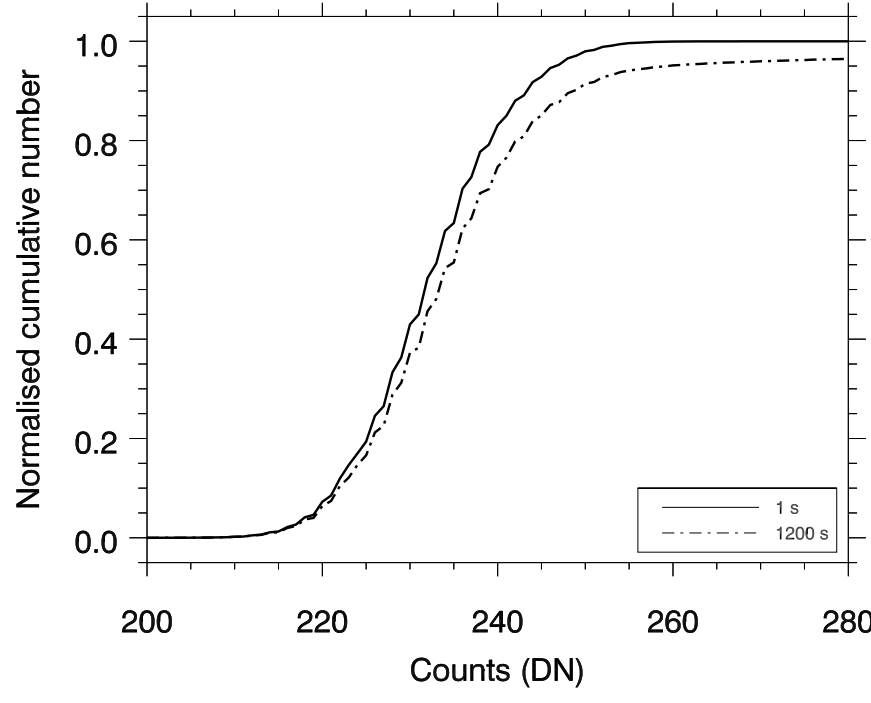

Fig. 3. Cumulative dark charge distribution as measured in a $1 \mathrm{~s}$ (solid curve) and a $1200 \mathrm{~s}$ (dot-dashed line) exposure dark image.

of the environment, thus the bias correction will become more significant.

Removal of coherent readout noise. The NAC and WAC signal chains are exposed to noise generated in the power converter modules of the CCD readout board (CRB) and the data processing unit (DPU). The periodic noise effects are in the 4-20 DN range. An effective noise reduction algorithm is currently under development, but is not implemented in the current version (therefore marked in gray in Fig. 2).

Dark current removal. A standard dark current subtraction is implemented in OsiCalliope.

Dark frames with exposure time between $1 \mathrm{~s}$ and $1200 \mathrm{~s}$ have been acquired to investigate the dark current behavior. Between May and December 2014, the CCD temperature was in the range $148-150 \mathrm{~K}$; the measured dark charge is $<0.006 \mathrm{e}^{-} / \mathrm{s}$, which corresponds to $<0.002 \mathrm{DN} / \mathrm{s}$. Even for long coma exposures (typically $\sim 300 \mathrm{~s}$ ) the dark current is much smaller than the readout noise at the current operational temperature; therefore, no dark current correction is applied.

Figure 3 shows the cumulative dark charge distribution as measured in a $1 \mathrm{~s}$ exposure and in a $1200 \mathrm{~s}$ exposure dark image. The distribution peaks around the bias level of 233 DN. A difference between the two distributions is present, indicating that a dark current is present in the $1200 \mathrm{~s}$ exposure, even though very small, on the order of $2.4 \mathrm{DN}$ in the $1200 \mathrm{~s}$ exposure.

High and low spatial frequency flat fielding. The flat field corrects for the nonuniform sensitivity of the camera across the image plane. Two main forms of nonuniformities are present: a) a pixel-to-pixel sensitivity variation across the image, due to the size difference and electrical characteristics of the individual CCD pixels and to dust particles on the CCD chip surface; b) a lower spatial frequency variation that is caused by the optical properties of the camera and the filters. To correct the OSIRIS images, we have introduced a new technique that consists in correcting the two types of nonuniformities in two steps. First the pixel-to-pixel sensitivity variations are removed and then the lower spatial frequency variations. However, as explained below, while the software is ready to handle the high- and lowfrequency flat fielding separately, the high- and low-frequency flat-fields themselves are not yet available.
Flat-fields cannot be acquired in flight, thus to calibrate the images we use flat-field frames acquired using the integrating sphere during the ground calibration of the OSIRIS cameras. A method for separating the high- and low-frequency contributions to the flat-fields is under development. Currently, the highspatial frequency flat fielding routine uses an artificial frame with constant intensity equal to 1 , while the low-spatial frequency routine uses the ground flat-field. Figure 4 shows examples of ground flat-field images for the NAC orange filter (left), the WAC OI filter (center), and the WAC OH filter (right). In the last image a pinhole is clearly visible and the pattern is the residual effect of the back thinning of the device and its amplitude is $\sim 1 \mathrm{DN}$.

Bad pixels and bad columns removal. Bad pixels are areas of the CCD surface that provide invalid DN values. They can be individual pixels, or groups of pixels (i.e., lines, rows, or areas). Once bad pixels have been identified, their location is marked and a correction is applied. Currently, the pipeline automatically corrects one bad column in the NAC and one cluster in the WAC. For the cluster, the correction is done by replacing the intensity of each pixel with the median of the intensities of the neighboring pixels. For the NAC bad column, the correction is done in two steps, since the first two neighboring columns are also problematic. First the average intensity of the neighboring columns is corrected by shifting the pixel values with the median difference of the adjacent good column. Then the intensity of the bad column is replaced with the median of the intensities of the neighboring pixels.

Ghosts and stray light correction. Ghosts are caused by parasitic reflections from the CCD and other optical components, such as the anti-radiation plate and the color filters. A deconvolution based correction method is under development, similar to the one applied in the Dawn camera (Kovacs et al. 2013). This step is currently marked in gray in Fig. 2.

Exposure time normalization. We divide each image by the mean effective exposure time. Because of the mechanical behavior of the shutter, we have to apply a correction to the commanded exposure time to determine the mean effective exposure time, which is the "real" time during which the CCD pixels see light. The exposure time correction is based on the actual movement of the shutter mechanism. During the exposure, the shutter shaft positions and times are recorded by high-resolution encoders and time counters. These data - the shutter pulses - are transmitted with each image, and are used to compute the mean effective exposure time. The mean effective exposure time varies slightly from line to line across the CCD, as shown in Fig. 5, where the mean effective exposure time for a commanded exposure of $0.33 \mathrm{~s}$ is plotted versus the CCD lines. This variation is less than $0.1 \mathrm{~ms}$ over the full CCD surface. On average, across the CCD the exposure time correction is $\sim 3 \mathrm{~ms}$, thus important for short nucleus exposures and less important when exposing for gas and dust.

Radiometric correction. To obtain flux calibrated images the conversion from $\mathrm{DN}$ to $\mathrm{W} / \mathrm{m}^{2} \mathrm{sr} \mathrm{nm}$ has to be applied. The absolute calibration coefficients are determined from observations of photometric standard stars. For this purpose we observed Vega, which has a high $\mathrm{S} / \mathrm{N}$ throughout the wavelength range spanned by the OSIRIS filters;16CygA+B, which is a solar-type star, but whose flux is too low in the UV range; and Zeta Cassiopeiae, a B-type star used to properly calibrate the UV range.

The raw level 1 star images are bias subtracted, flat fielded and divided by the exposure time. After the center of the star is identified, its flux is measured with the technique of aperture photometry. The tabulated flux of the star at the central 

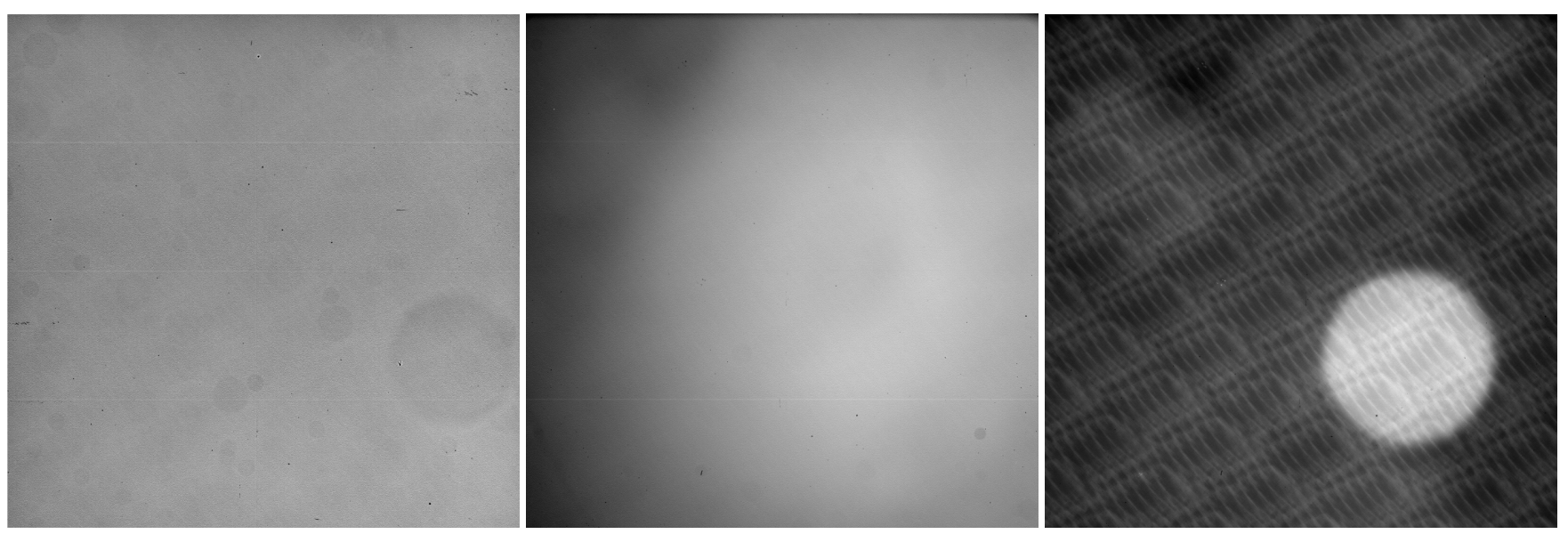

Fig. 4. Example of ground flat-field frames used to calibrate the images. Left: flat-fields for NAC orange images. Center: flat-field for WAC OI images. Right: Flat-field for WAC OH images. In this last image a pinhole is clearly visible.

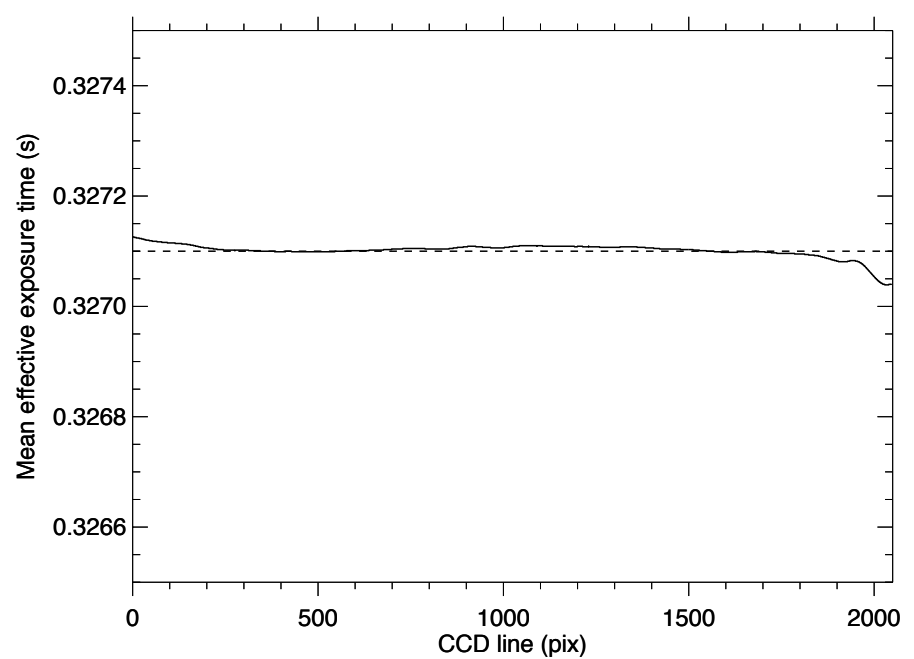

Fig. 5. Mean effective exposure time for a commanded exposure of $0.33 \mathrm{~s}$ across the full CCD surface, as measured based on shutter pulse data.

wavelength of each filter (in $\mathrm{W} / \mathrm{m}^{2} \mathrm{sr} \mathrm{nm}$ ) is determined from its tabulated magnitude. The absolute calibration coefficients are defined as

$A=\frac{\text { flux }_{\text {measured }}}{\text { flux }_{\text {tabulated }} \times k^{-1}}$

where $k$ is the pixel scale of the camera (in sr).

If the photometric standard star is not a solar-type star, as in the case of Vega, the calibration constant must be scaled to solar colors.

To radiometrically calibrate OSIRIS data acquired posthibernation, images of Vega acquired in May 2014 were used. However, due to shutter failures, for certain filter combinations, no new images were successfully acquired in May 2014, thus the absolute calibration coefficients determined pre-hibernation were used. However, new images of Vega were acquired afterward to determine the missing absolute calibration coefficients and to check the stability over time of the absolute calibration.

The absolute calibration coefficients are calculated for the central wavelength of the corresponding filter. The uncertainty associated with each absolute calibration coefficient only takes the aperture photometry error into account. Other contributions, such as the uncertainty on the CCD quantum efficiency, on the filter transmission curves, on the reflectivity of the mirrors, and

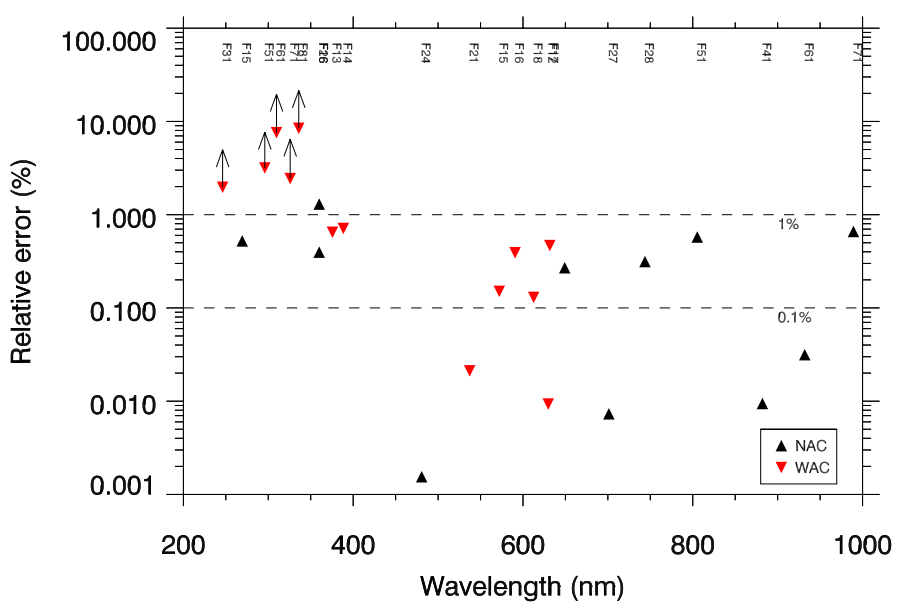

Fig. 6. Relative errors of the absolute calibration coefficients versus wavelength. The WAC UV filters that are affected by pinholes, which effectively decrease the accuracy of the radiometric calibration, are marked by a black arrow to indicate that the relative error is larger than the value shown.

on the position of the central wavelength of the filters are not included at this point, thus the determined uncertainty and relative error are lower limits. The relative errors of the absolute calibration coefficients versus wavelength are shown in Fig. 6. The absolute calibration coefficients for all NAC filters (black triangles in Fig. 6) and WAC filters (red triangle in Fig. 6) at wavelength $\lambda \geq 375 \mathrm{~nm}$ have a relative uncertainty $\leq 1 \%$. The WAC filters at $\lambda \leq 375 \mathrm{~nm}$ have very narrow bandpass and transmission $\leq 0.4$ (see Fig. 1). The Vega spectrum has low flux in the UV wavelength range that, combined with the transmission curves of the WAC UV filters, results in low S/N photometric standard star images in the considered wavelength range. This translates into high relative errors of the absolute calibration coefficients, as shown in Fig. 6. To improve the absolute calibration in the UV wavelength range we also observed Zeta Cassiopeiae, a B-type star with high flux and smooth spectrum in the UV wavelength range.

The WAC UV filters are affected by pinholes, manufacturing defects (small holes in the filters) that allow light out of the selected wavelength band to pass through. Pinholes effectively decrease the accuracy of the radiometric calibration for those filters. A description of the radiometric calibration of OSIRIS 
images is presented by Magrin et al. (2015). They used a different method from the one used by the pipeline and described in this work. Moreover, their absolute calibration coefficients are obtained from photometric standard star images acquired before hibernation, thus a direct comparison between their results and those described here is not straightforward.

Geometric distortion correction. NAC and WAC optical layouts are off-axis mirror systems, which provide high transmittance from the UV to the near-IR and diffraction limited performance with low geometrical optical aberrations. However, this layout has a significant geometrical distortion that must be corrected. The correction is performed by resampling the images according to the nonlinear distortion function of the camera, as if it had been acquired by a distortion-free camera. The image resampling is done by the bi-linear algorithm, and since the original image is in radiance units, the result is also considered radiometrically corrected on large scales. The resampling is based on a $2 \mathrm{D}$ third-order polynomial fit,

$$
\begin{aligned}
X_{i} & =\sum_{i, j} k x_{i, j} \cdot X_{\mathrm{o}}{ }^{j} \cdot Y_{\mathrm{o}}{ }^{i}, \\
Y_{i} & =\sum_{i, j} k y_{i, j} \cdot X_{\mathrm{o}}{ }^{j} \cdot Y_{\mathrm{o}}{ }^{i},
\end{aligned}
$$

where $\left(X_{i}, Y_{i}\right)$ are the undistorted coordinates expressed as functions of the original coordinates $\left(X_{0}, Y_{\mathrm{o}}\right)$ and $k x$ and $k y$ are the coefficients for the distortion removal.

During the ground calibration of OSIRIS, the distortion correction coefficients were measured, taking images of a metallic grid of 73 pinholes. During in-flight calibration campaigns, star field images were acquired to check and improve the quality of the geometric distortion correction. We have estimated that WAC distortion corrected images (level 3) have an accuracy in the position of about 1-1.5 pix (Naletto et al. 2002). The accuracy in the position for the NAC images is 0.1 pixel and maximum error in the FOV is 0.5 pix.

The amplitude of the geometric distortion is about 15 pix for the NAC and 80 pix for the WAC in a corner of the level 2 images. For both cameras, we determined the coefficients of the polynomial functions which allow the $(X, Y)$ pixel coordinates in level 2 images to be converted into coordinates in level 3 images (and vice versa). We fitted the coefficients using several star fields typically containing 5000 stars whose equatorial coordinates were taken from the USNO catalog.

The effect of the geometric distortion is nicely illustrated in Fig. 7 where the contour of a distorted (level 2) image is superimposed on a geometric distortion corrected (level 3) image.

Ghosts and stray light removal remain an open point. Future campaigns are planned to acquire the data that are needed to address and properly correct these issues.

In addition to the radiometrically calibrated and distortion corrected frame, the pipeline creates an error map and a quality map for each image. The error map contains the error associated with the intensity of each pixel, calculated using the Poisson statistics and the (bias) readout noise error. The pixel quality map is an 8-bit image with encoded quality indicators of each pixel. These include bad pixels, saturation, nonlinearity range, warm and cold pixels, and lossy compression.

\section{Results: instrument science}

Here we want to address the overall accuracy that we can expect in our scientific results based on the accuracy of the calibration that we have performed. This is not meant to replace a full error

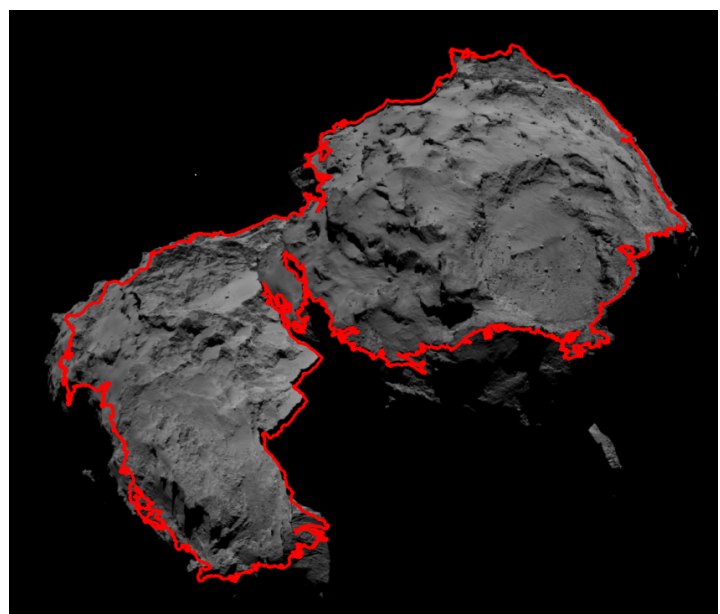

Fig. 7. Effect of the geometric distortion correction on a WAC image. The contour of a distorted image is superimposed on a geometric distortion corrected image.

analysis of the specific works carried out, but as a general guide to the level of trust that can be put into various analyses.

Shape reconstruction. A key contribution to the accuracy of the shape reconstruction using stereo-photogrammetry (SPG) comes from the accuracy of the geometric distortion correction. Any distortion error of a few tenths of a pixel may cause errors in the stereo-derived 3D information up to a pixel or more, depending on the kind of error and on the stereo geometry (small stereo angles cause higher errors). For photoclinometry, errors in both the geometric and radiometric calibration can lead to incorrect slopes, and thus to inaccuracies in the coordinates of the vertices of the final reconstructed models. The link between such errors and the errors in slope is the subject of further investigations.

Geomorphology. The geographical and geological definition of the cometary regions does not closely rely on a specific calibration of the images since they are based on regional and local mutual limits that can even be recognized on level 2 images. The only effect that level 2 images have on such studies is the possible stretch of the recognized areas, due to image deformation, with respect to the real ones. Nevertheless, the possibility of working on distortion corrected images is of paramount importance when statistical studies need to be performed on the surface, especially for small (a few pixels in width) features such as boulders, pits, fractures, layering, and terrace margins in addition to the detection of surface changes due to localized activity. Statistical and quantitative studies involving the physical dimensions of any of these fine features (e.g., boulders) or their lateral spacing (e.g., layers or fracture systems) would not have the desired or needed accuracy without a distortion-free database.

Composition of the nucleus and dust. The photometric accuracy, expressed in terms of uncertainty of $I / F$, has different contributions: (i) the uncertainty of the measured star flux from aperture photometry, which is usually small, since the $\mathrm{S} / \mathrm{N}$ of the photometric standard star images is very high; (ii) the accuracy of the reference star spectrum and of the solar spectrum used in the determination of the absolute calibration coefficients; (iii) uncertainty of the central wavelength of each filter, particularly critical for the UV region; (iv) pinhole presence in some filters; and (v) stray light contribution for different observing geometries. Taking into account all these contributions, we estimated $I / F$ uncertainties of $\sim 4-6 \%$ for the NAC UV filters, $\sim 8-10 \%$ for the WAC UV filters, and $\sim 20-25 \%$ for the WAC far-UV filters. The visible and near-IR have a much higher accuracy, 
$\sim 1-1.7 \%$ for both cameras, thanks to cross-camera calibration and observations of Vesta performed before Rosetta entered hibernation (Fornasier et al. 2011).

Dust coma and dust grains. Studies of the dust coma environment and grains in the inner coma require high-accuracy geometric distortion correction and radiometric calibration of the images. An unresolved coma was detected around the nucleus in the earliest images in March 2014, while individual grains were identified in OSIRIS images of the inner coma from September 2014 onward. The determination of grain orbits allows them to be used as test particles in understanding the complex comet gravitational field. Their photometric measurements provide hints on the size and composition of the materials released from the comet because of the action of the sublimation of volatiles. However, an important step in dust grain studies is the star background removal, which is obtained by co-registering consecutive images on stars. The discrepancy between the measured position of a star and the expected position from astrometric catalogs in geometric distortion corrected images has different contributions: a) the accuracy of the geometric distortion correction; b) the intrinsic errors in the astrometric catalog position; c) the precision of the software used in recognizing the CCD location of the star's optocenter with a 2D Gaussian fit of the objects PSF, and d) the possible proper motion of the stars between the catalog date and the date of observations. The accuracy on the star position was determined to be 5 pixels for the NAC images (Bertini et al. 2015) and 3 pixels for the WAC images (Davidsson et al. 2015). This result emphasizes that the major contribution to the position accuracy is not given by the accuracy of the distortion correction. However, an inaccurate correction of the geometric distortion would translate into a larger error on the image co-registration with possible misinterpretations in the stellar background removal.

The photometric accuracy affects the grain photometry in the same way as it affects the nucleus photometry, as was described in the previous section.

Gas distribution and production rates. From the analysis of gas images acquired at the end of December 2014, when the comet was about 2.6 AU from the Sun, we concluded that the bias and its uncertainty are small contributors $(\leq 0.1 \%$ for OI images) to the final accuracy of the gas production rates. A much larger contribution comes from the continuum removal factor, known with an accuracy of $1-10 \%$ depending on which filter pair is considered. New observations of $16 \mathrm{CygA}+\mathrm{B}$ that are planned for the coming months will help to improve the accuracy in the determination of the continuum removal factor, which would translate into more accurate gas production rates. From the analysis of gas images acquired at the end of December 2014, when the comet was about 2.6 AU from the Sun, we concluded that the bias and its uncertainty are small contributors $(\leq 0.1 \%$ for OI images) to the final accuracy of the gas production rates. A much larger contribution comes from the continuum removal factor, known with an accuracy of 1-10\% depending on which filter pair is considered. New observations of $16 \mathrm{CygA}+\mathrm{B}$ that are planned for the coming months will help to improve the accuracy in the determination of the continuum removal factor, which would translate into more accurate gas production rates. The continuum subtracted images in the OI filter has a $S / N \sim 70$ near the limb and $\sim 40$ near the edge of the frame. The $\mathrm{S} / \mathrm{N}$ in the UV filters is lower: the $\mathrm{OH}$ filter has a $S / N \sim 20$ near the limb and $\sim 5$ near the edge of the frame.

A major uncertainty in the determination of the gas production rates is the contamination from different gas species. An additional source of uncertainty comes from pinholes and stray

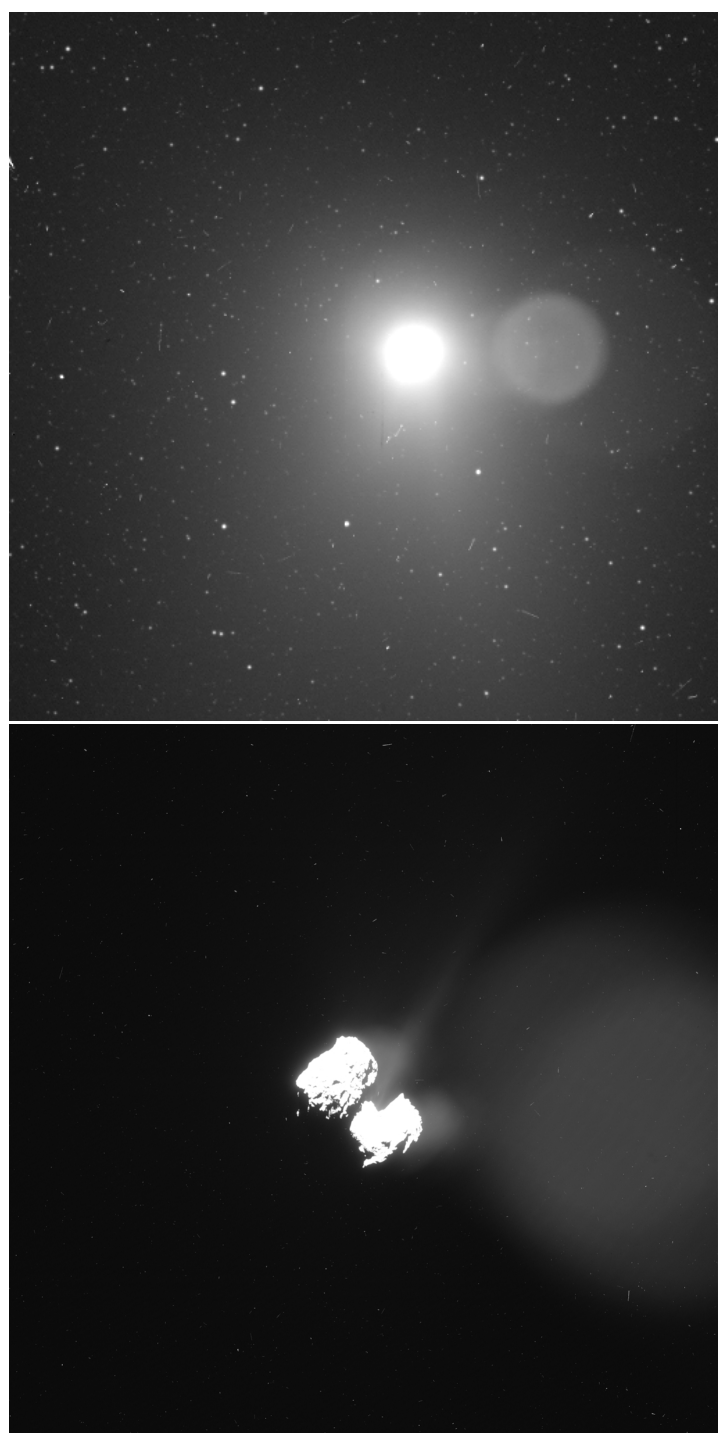

Fig. 8. Top panel: image of $67 \mathrm{P}$ acquired on July 25, 2014. A ghost is clearly visible on the right-hand side of the image. Bottom panel: image acquired on August 8, 2014. The ghost is in the same position as in the top panel and it is blending with the coma, creating artificial structures.

light. An investigation into quantifying their contribution to the determination of gas production rates is ongoing. Care should be taken owing to the presence of ghosts in the images. Figure 8 (top panel) shows an image of the comet acquired on July 25, 2014, when Rosetta was $\sim 3000 \mathrm{~km}$ from the comet. A ghost is clearly visible on the right-hand side of the image. In Fig. 8 (bottom panel) the ghost is in the same position as in the top panel, and it is immediately clear that the ghost is blending with the coma, creating artificial structures. No ghost correction is applied to the data and extra care should be taken while analyzing the data. However, it should be noted that since the ghost comes from internal reflections, it is therefore always located in the same place, which allows us to reliably identify it.

Jets. Figure 9 shows faint jets arising from the surface of the comet. The geometric distortion correction affects the tracking of jet footprints. The accuracy on the photometric profile depends on the radiometric calibration of the images, stray light, and ghosts. 


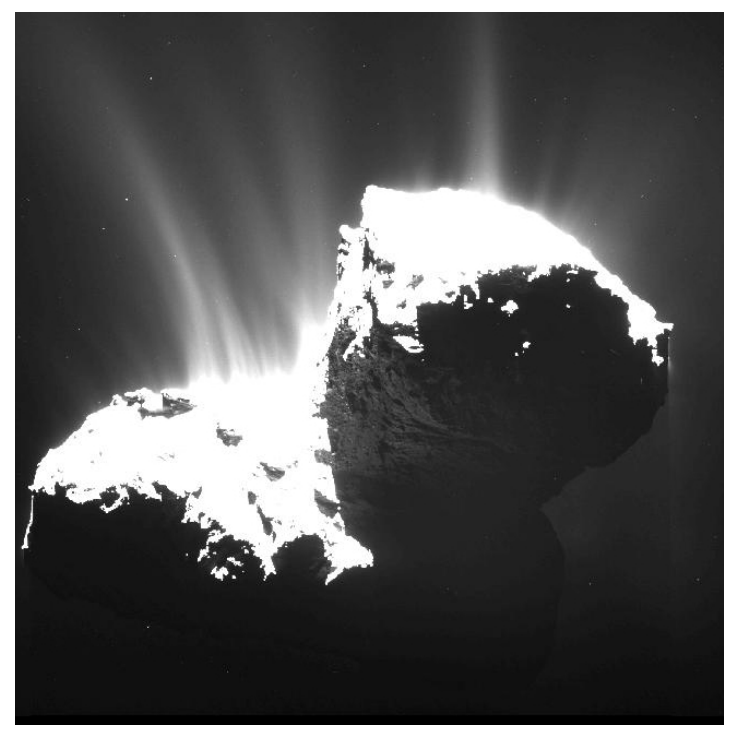

Fig. 9. Image of 67P acquired on November 22, 2014, from a distance of $30 \mathrm{~km}$ from the comet. The nucleus is overexposed to show faint jets.

\section{Summary}

We have described the pipeline that is used to calibrate OSIRIS images and how each of the parameters used in the calibration process has been determined. We estimated the accuracy that we can expect in our scientific results based on the calibration accuracy, providing a level of trust that can be used in the different analyses performed with OSIRIS images. Open issues are still present, such as ghost and stray light removal or higher accuracy radiometric calibration in the UV wavelength range. Future calibration campaigns are planned to acquire the data that are needed to address and properly correct these issues.

Acknowledgements. OSIRIS was built by a consortium led by the Max-PlanckInstitut für Sonnensystemforschung, Göttingen, Germany, in collaboration with CISAS, University of Padova, Italy, the Laboratoire d'Astrophysique de Marseille, France, the Instituto de Astrofísica de Andalucia, CSIC, Granada, Spain, the Scientific Support Office of the European Space Agency, Noordwijk, The Netherlands, the Instituto Nacional de Técnica Aeroespacial, Madrid, Spain, the Universidad Politéchnica de Madrid, Spain, the Department of Physics and Astronomy of Uppsala University, Sweden, and the Institut für Datentechnik und Kommunikationsnetze der Technischen Universität Braunschweig, Germany. The support of the national funding agencies of Germany (DLR), France (CNES), Italy (ASI), Spain (MEC), Sweden (SNSB), and the ESA Technical
Directorate is gratefully acknowledged. We thank the Rosetta Science Ground Segment at ESAC, the Rosetta Mission Operations Centre at ESOC and the Rosetta Project at ESTEC for their outstanding work enabling the science return of the Rosetta Mission.

\section{Appendix A: The OSIRIS data levels}

The OSIRIS data levels correspond to CODMAC (Committee on Data Management, Archiving, and Computing) levels: OSIRIS level $n=$ CODMAC level $n+1$.

- Level 0: PDS image format, raw header (proprietary, nonstandard labels), raw binary with 16-bit integer image data.

- Level 1: PDS format, calibrated header (standard labels), raw binary with 16-bit integer image data.

- Level 2: PDS format, calibrated header, radiometric calibrated 32-bit float image data, in $\mathrm{W} / \mathrm{m}^{2} \mathrm{sr} \mathrm{nm}$ units.

- Level 3: PDS format, calibrated header, radiometric calibrated 32-bit float image data, corrected for the camera optical distortion, in $\mathrm{W} / \mathrm{m}^{2}$ sr nm units.

- Level 3B: PDS format, calibrated header, radiometric calibrated 32-bit float image data, corrected for the camera optical distortion in reflectance units.

\section{References}

Auger, A.-T., Groussin, O., Jorda, L., et al. 2015, A\&A, 583, A35 Bertini, I., Thomas, N., \& Barbieri, C. 2007, A\&A, 461, 351

Bertini, I., Gutiérrez, P. J., Lara, L. M., et al. 2015, A\&A, 583, A19

Davidsson, B., Gutiérrez, P. J., Sierks, H., et al. 2015, A\&A, 583, A16

El-Maarry, M. R., Thomas, N., Giacomini, L., et al. 2015, A\&A, 583, A26

Fornasier, S., Mottola, S., Barucci, M. A., Sierks, H., \& Hviid, S. 2011, A\&A, 533, L9

Fornasier, S., Hasselmann, P. H., Barucci, M. A., et al. 2015, A\&A, 583, A30

Keller, H. U., Barbieri, C., Lamy, P., et al. 2007, Space Sci. Rev., 128, 433

Kovacs, G., Sierks, H., Nathues, A., Richards, M., \& Gutierrez-Marques, P. 2013, in SPIE Conf. Ser., 8889, 12

Lacerda, P., \& Jewitt, D. 2012, ApJ, 760, L2

Lin, Z.-Y., Ip, W.-H., Lai, I.-L., et al. 2015, A\&A, 583, A11

Magrin, S., La Forgia, F., Da Deppo, V., et al. 2015, A\&A, 574, A123

Massironi, M., Simioni, E., Marzari, F., et al. 2015, Nature, accepted

Naletto, G., DaDeppo, V., Pelizzo, M. G., Ragazzoni, R., \& Marchetti, E. 2002, Appl. Opt., 41, 1446

Pajola, M., Vincent, J.-B., Güttler, C., et al. 2015, A\&A, 583, A37

Pommerol, A., Thomas, N., El-Maarry, M. R., et al. 2015, A\&A, 583, A25

Rotundi, A., Sierks, H., Della Corte, V., et al. 2015, Science, 347, 3905

Thomas, N., Sierks, H., Barbieri, C., et al. 2015, Science, 347, 440

Tubiana, C., Snodgrass, C., Bertini, I., et al. 2015, A\&A, 573, A62 